\title{
SHARP UPPER BOUNDS FOR FRACTIONAL MOMENTS OF THE RIEMANN ZETA FUNCTION
}

\author{
WINSTON HEAP, MAKSYM RADZIWIEE, AND K. SOUNDARARAJAN
}

\begin{abstract}
We establish sharp upper bounds for the $2 k$ th moment of the Riemann zeta function on the critical line, for all real $0 \leqslant k \leqslant 2$. This improves on earlier work of Ramachandra, Heath-Brown and Bettin-Chandee-Radziwiłł.
\end{abstract}

\section{INTRODUCTION}

This paper is concerned with the moments of the Riemann zeta function on the critical line: namely, with the quantity

$$
I_{k}(T)=\int_{T}^{2 T}\left|\zeta\left(\frac{1}{2}+i t\right)\right|^{2 k} d t
$$

where $k>0$ is real and $T$ is large. The problem of understanding the behavior of these moments is central in the theory of the Riemann zeta-function. The classical work of Hardy and Littlewood [6], and Ingham [8] established asymptotic formulae for $I_{k}(T)$ in the cases $k=1$ and 2 , and these still remain the only situations where an asymptotic is known. Lacking an asymptotic, much work has been focussed on the problems of obtaining sharp upper and lower bounds for these moments. Lower bounds of the form $I_{k}(T) \gg_{k} T(\log T)^{k^{2}}$ are established for all $k \geqslant 1$ in Radziwiłł and Soundararajan [9] unconditionally, and for all $k \geqslant 0$ conditionally on the Riemann Hypothesis in papers of Heath-Brown and Ramachandra, see [11, 12, 5]. Upper bounds of the form $I_{k}(T) \ll_{k} T(\log T)^{k^{2}}$ are known when $k=1 / n$ for natural numbers $n$ (due to Heath-Brown [5]) and when $k=1+1 / n$ for natural numbers $n$ (by work of Bettin, Chandee, and Radziwitł [2]). Conditionally on the Riemann Hypothesis, the work of Harper [4], refining earlier work of Soundararajan [13], establishes that $I_{k}(T) \ll_{k} T(\log T)^{k^{2}}$ for all $k \geqslant 0$. This paper adds to our knowledge on moments by establishing a sharp upper bound for $I_{k}(T)$ for all real $0 \leqslant k \leqslant 2$.

The first author is supported by European Research Council grant no. 670239. The second author acknowledges the support of a Sloan fellowship. The third author is partially supported by a grant from the National Science Foundation, and by a Simons Investigator grant from the Simons Foundation. 
Theorem 1. Let $0 \leqslant k \leqslant 2$. Then, for $T \geqslant 10$,

$$
I_{k}(T) \ll T(\log T)^{k^{2}} .
$$

The proof of the theorem is based on the method introduced in Radziwiłl and Soundararajan [10] which enunciates that if in a family of $L$-values, asymptotics for a particular moment can be established with a little room to spare, then sharp upper bounds may be obtained for all smaller moments. Theorem 1 is an illustration of this principle, and combines the ideas of [10] together with knowledge of the fourth moment of $\zeta(s)$ twisted by short Dirichlet polynomials (see the work of Hughes and Young [7], and Betin, Bui, Li, and Radziwiłł [1]).

\section{Plan of the Proof of Theorem 1}

Throughout, $\log _{j}$ will denote the $j$-fold iterated logarithm. Let $T$ be large, and let $\ell$ denote the largest integer such that $\log _{\ell} T \geqslant 10^{4}$. Define a sequence $T_{j}$ by setting $T_{1}=e^{2}$, and for $2 \leqslant j \leqslant \ell$ by

$$
T_{j}:=\exp \left(\frac{\log T}{\left(\log _{j} T\right)^{2}}\right) .
$$

Note that $e^{2}=T_{1}<T_{2}<\ldots<T_{\ell} \leqslant T^{10^{-8}}$.

For each $2 \leqslant j \leqslant \ell$, set

$$
\mathcal{P}_{j}(s):=\sum_{T_{j-1} \leqslant p<T_{j}} \frac{1}{p^{s}}, \quad \text { and } \quad P_{j}=\mathcal{P}_{j}(1)=\sum_{T_{j-1} \leqslant p<T_{j}} \frac{1}{p} .
$$

Note that for large $T$,

$$
P_{j} \sim \log \frac{\log T_{j}}{\log T_{j-1}}=2 \log \left(\frac{\log _{j-1} T}{\log _{j} T}\right)=2 \log _{j} T-2 \log _{j+1} T,
$$

so that $P_{\ell} \geqslant 10^{4}, P_{\ell-1} \geqslant \exp \left(10^{4}\right)$, and so on. Further, define

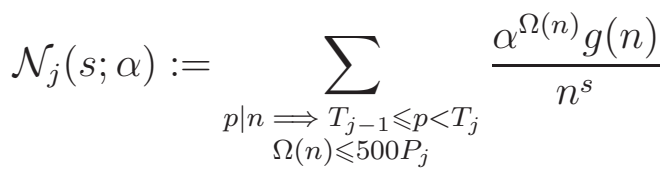

where $g(n)$ denotes the multiplicative function given on prime powers by $g\left(p^{r}\right)=1 / r$ !

The motivation for these definitions is the following. Typically one might expect that $\zeta\left(\frac{1}{2}+i t\right)^{\alpha}$ is similar to $\prod_{j \leqslant \ell} \exp \left(\alpha \mathcal{P}_{j}\left(\frac{1}{2}+i t\right)\right)$. Now most of the time, $\left|\mathcal{P}_{j}\left(\frac{1}{2}+i t\right)\right|$ is no more than $50 P_{j}$, in which case by a Taylor approximation one can approximate $\exp \left(\alpha \mathcal{P}_{j}\left(\frac{1}{2}+i t\right)\right)$ by $\mathcal{N}_{j}\left(\frac{1}{2}+i t ; \alpha\right)$ (see Lemma 1 below). Thus, for most $t$ we shall be able to replace $\zeta\left(\frac{1}{2}+i t\right)^{\alpha}$ by $\prod_{j \leqslant \ell} \mathcal{N}_{j}\left(\frac{1}{2}+i t ; \alpha\right)$, which is a short Dirichlet polynomial (of length $\leqslant T^{1 / 10}$, say) and thus facilitates computations. 
We now state three propositions from which the main theorem will follow, postponing the proofs of the propositions to later sections.

Proposition 1. Let $0 \leqslant k \leqslant 2$ be a given real number. Then, for all complex numbers $s$ inside the critical strip $0<$ Re $s<1$,

$$
\begin{aligned}
|\zeta(s)|^{2 k} & \leqslant k|\zeta(s)|^{4} \prod_{2 \leqslant j \leqslant \ell}\left|\mathcal{N}_{j}(s ; k-2)\right|^{2}+(2-k) \prod_{2 \leqslant j \leqslant \ell}\left|\mathcal{N}_{j}(s ; k)\right|^{2} \\
& +\sum_{2 \leqslant v \leqslant \ell}\left(k|\zeta(s)|^{4} \prod_{2 \leqslant j<v}\left|\mathcal{N}_{j}(s, k-2)\right|^{2}+(2-k) \prod_{2 \leqslant j<v}\left|\mathcal{N}_{j}(s ; k)\right|^{2}\right)\left|\frac{\mathcal{P}_{v}(s)}{50 P_{v}}\right|^{2\left\lceil 50 P_{v}\right\rceil} .
\end{aligned}
$$

Proposition 2. Let $0 \leqslant k \leqslant 2$ real, be given. Then

$$
\int_{T}^{2 T} \prod_{2 \leqslant j \leqslant \ell}\left|\mathcal{N}_{j}\left(\frac{1}{2}+i t ; k\right)\right|^{2} d t \ll T(\log T)^{k^{2}}
$$

and for all $2 \leqslant v \leqslant \ell$ and $0 \leqslant r \leqslant\left\lceil 50 P_{v}\right\rceil$,

$$
\int_{T}^{2 T} \prod_{2 \leqslant j<v}\left|\mathcal{N}_{j}\left(\frac{1}{2}+i t ; k\right)\right|^{2}\left|\mathcal{P}_{v}\left(\frac{1}{2}+i t\right)\right|^{2 r} d t \ll T\left(\log T_{v-1}\right)^{k^{2}}\left(r ! P_{v}^{r}\right) .
$$

Proposition 3. Let $0 \leqslant k \leqslant 2$ real, be given. Then

$$
\int_{T}^{2 T}\left|\zeta\left(\frac{1}{2}+i t\right)\right|^{4} \prod_{2 \leqslant j \leqslant \ell}\left|\mathcal{N}_{j}\left(\frac{1}{2}+i t ; k-2\right)\right|^{2} d t \ll T(\log T)^{k^{2}},
$$

and for all $2 \leqslant v \leqslant \ell$ and $0 \leqslant r \leqslant\left\lceil 50 P_{v}\right\rceil$,

$$
\begin{aligned}
\int_{T}^{2 T}\left|\zeta\left(\frac{1}{2}+i t\right)\right|^{4} & \prod_{2 \leqslant j<v}\left|\mathcal{N}_{j}\left(\frac{1}{2}+i t ; k-2\right)\right|^{2}\left|\mathcal{P}_{v}\left(\frac{1}{2}+i t\right)\right|^{2 r} d t \\
& \ll T(\log T)^{4}\left(\log T_{v-1}\right)^{k^{2}-4}\left(18^{r} r ! P_{v}^{r} \exp \left(P_{v}\right)\right)
\end{aligned}
$$

We quickly deduce Theorem 1 from the above propositions.

Proof of Theorem 1. Combining the above propositions we find

$$
\begin{aligned}
\int_{T}^{2 T}\left|\zeta\left(\frac{1}{2}+i t\right)\right|^{2 k} d t & \ll T(\log T)^{k^{2}}+\sum_{2 \leqslant v \leqslant \ell} T\left(\log T_{v-1}\right)^{k^{2}} \\
& \times\left(\frac{\left\lceil 50 P_{v}\right\rceil ! P_{v}^{\left\lceil 50 P_{v}\right\rceil}}{\left(50 P_{v}\right)^{2\left\lceil 50 P_{v}\right\rceil}}+\left(\frac{\log T}{\log T_{v-1}}\right)^{4} \frac{18^{\left\lceil 50 P_{v}\right\rceil}\left\lceil 50 P_{v}\right\rceil ! P_{v}^{\left\lceil 50 P_{v}\right\rceil} \exp \left(P_{v}\right)}{\left(50 P_{v}\right)^{2\left\lceil 50 P_{v}\right\rceil}}\right) .
\end{aligned}
$$


A quick calculation shows that the above is

$$
\begin{aligned}
& \ll T(\log T)^{k^{2}}\left(1+\sum_{2 \leqslant v \leqslant \ell}\left(\frac{\log T}{\log T_{v-1}}\right)^{4} \exp \left(-50 P_{v}\right)\right) \\
& \ll T(\log T)^{k^{2}}\left(1+\sum_{2 \leqslant v \leqslant \ell}\left(\log _{v-1} T\right)^{8}\left(\frac{\log _{v} T}{\log _{v-1} T}\right)^{100}\right) \ll T(\log T)^{k^{2}} .
\end{aligned}
$$

\section{Proof of Proposition 1}

Lemma 1. Let $|\alpha| \leqslant 2$ be a real number, $T$ be sufficiently large, and $s$ be a complex number. For all $2 \leqslant j \leqslant \ell$, if $\left|\mathcal{P}_{j}(s)\right| \leqslant 50 P_{j}$ then

$$
\exp \left(2 \alpha \operatorname{Re} \mathcal{P}_{j}(s)\right) \leqslant\left(1-e^{-P_{j}}\right)^{-1}\left|\mathcal{N}_{j}(s ; \alpha)\right|^{2} .
$$

Proof. Expanding $\exp \left(\alpha \mathcal{P}_{j}(s)\right)$ using a Taylor series, and using the assumption $\left|\mathcal{P}_{j}(s)\right| \leqslant$ $50 P_{j}$, we find that

$$
\left|\exp \left(\alpha \mathcal{P}_{j}(s)\right)\right| \leqslant\left|\sum_{m \leqslant 500 P_{j}} \frac{\alpha^{m} \mathcal{P}_{j}(s)^{m}}{m !}\right|+2 \cdot \frac{\left(100 P_{j}\right)^{500 P_{j}}}{\left\lceil 500 P_{j}\right\rceil !} .
$$

The last term is $\leqslant e^{-250 P_{j}}$, while $\left|\exp \left(\alpha \mathcal{P}_{j}(s)\right)\right| \geqslant \exp \left(-|\alpha| 50 P_{j}\right) \geqslant \exp \left(-100 P_{j}\right)$. Therefore, since $P_{j} \geqslant 10^{4}$, we may easily conclude that

$$
\left|\exp \left(\alpha \mathcal{P}_{j}(s)\right)\right|^{2} \leqslant\left(1-e^{-P_{j}}\right)^{-1}\left|\sum_{m \leqslant 500 P_{j}} \frac{\alpha^{m} \mathcal{P}_{j}(s)^{m}}{m !}\right|^{2} .
$$

Since

$$
\frac{\mathcal{P}_{j}(s)^{m}}{m !}=\frac{1}{m !} \sum_{T_{j-1} \leqslant p_{1}, \ldots, p_{m}<T_{j}} \frac{1}{\left(p_{1} \ldots p_{m}\right)^{s}}=\sum_{\substack{\Longrightarrow \mid n \\ \Rightarrow(n)=1 \leqslant p<T_{j} \\ \Omega(n)=m}} \frac{g(n)}{n^{s}}
$$

the proposition follows.

Proof of Proposition 1. This proposition is an analogue of Lemma 2 of [10], and is proved similarly. We make use of Young's inequality $a b \leqslant a^{p} / p+b^{q} / q$ for any nonnegative real numbers $a$ and $b$, and non-negative $p$ and $q$ with $1 / p+1 / q=1$.

If $\left|\mathcal{P}_{j}(s)\right| \leqslant 50 P_{j}$ for all $2 \leqslant j \leqslant \ell$ then using Young's inequality with $p=4 / 2 k$ and $q=4 /(4-2 k)$ we have

$$
|\zeta(s)|^{2 k} \leqslant \frac{k}{2}|\zeta(s)|^{4} \prod_{2 \leqslant j \leqslant \ell} e^{(-4+2 k) \operatorname{Re} \mathcal{P}_{j}(s)}+\left(1-\frac{k}{2}\right) \prod_{2 \leqslant j \leqslant \ell} e^{2 k \operatorname{Re} \mathcal{P}_{j}(s)}
$$


By Lemma 1 the right hand side is

$$
\leqslant \prod_{2 \leqslant j \leqslant \ell}\left(1-e^{-P_{j}}\right)^{-1}\left(\frac{k}{2}|\zeta(s)|^{4} \prod_{2 \leqslant j \leqslant \ell}\left|\mathcal{N}_{j}(s ; k-2)\right|^{2}+\left(1-\frac{k}{2}\right) \prod_{2 \leqslant j \leqslant \ell}\left|\mathcal{N}_{j}(s ; k)\right|^{2}\right) .
$$

Since $\prod_{2 \leqslant j \leqslant \ell}\left(1-e^{-P_{j}}\right)^{-1} \leqslant 2$, this contribution is bounded by the first two terms in the proposition.

Now suppose that there exists an integer $2 \leqslant v \leqslant \ell$ for which $\left|\mathcal{P}_{j}(s)\right| \leqslant 50 P_{j}$ whenever $2 \leqslant j<v$, but with $\left|\mathcal{P}_{v}(s)\right|>50 P_{v}$. Then applying Young's inequality and Lemma 1 as before, and noting that $\left|\mathcal{P}_{v}(s)\right| /\left(50 P_{v}\right) \geqslant 1$, we find

$$
|\zeta(s)|^{2 k} \leqslant\left(k|\zeta(s)|^{4} \prod_{2 \leqslant j<v}\left|\mathcal{N}_{j}(s ; k-2)\right|^{2}+(2-k) \prod_{2 \leqslant j<v}\left|\mathcal{N}_{j}(s ; k)\right|^{2}\right)\left|\frac{\mathcal{P}_{v}(s)}{50 P_{v}}\right|^{2\left\lceil 50 P_{v}\right\rceil} .
$$

Summing this over all $2 \leqslant v \leqslant \ell$, we obtain Proposition 1 .

\section{Proof of Proposition 2}

We give a proof of the second assertion of the proposition, the first statement being similar. Since $\prod_{2 \leqslant j<v} \mathcal{N}_{j}(s ; k) \mathcal{P}_{v}(s)^{r}$ is a Dirichlet polynomial of length $\leqslant T^{1 / 10}$, using the familiar mean value estimate for Dirichlet polynomials, we find that

$$
\begin{aligned}
\int_{T}^{2 T} & \prod_{2 \leqslant j<v}\left|\mathcal{N}_{j}(s ; k)\right|^{2}\left|\mathcal{P}_{v}(s)\right|^{2 r} d t \\
& \ll T \prod_{2 \leqslant j<v}\left(\sum_{\substack{p \mid n_{j} \\
\Omega\left(n_{j}\right) \leqslant 500 P_{j}}} \frac{k^{2 \Omega\left(n_{j}\right)}}{n_{j}}\right) \sum_{\substack{p<T_{j} \\
\Longrightarrow T_{v-1} \leqslant p<T_{v} \\
\Omega(n)=r}} \frac{(r ! g(n))^{2}}{n} .
\end{aligned}
$$

Now note that,

$$
\sum_{p \mid n_{j} \Longrightarrow T_{j-1} \leqslant p<T_{j}} \frac{k^{2 \Omega\left(n_{j}\right)}}{n_{j}} \leqslant \prod_{T_{j-1} \leqslant p<T_{j}}\left(1+\frac{k^{2}}{p}+\frac{k^{4}}{p^{2}}+\ldots\right) \ll\left(\frac{\log T_{j}}{\log T_{j-1}}\right)^{k^{2}},
$$

where we used that $p \geqslant T_{1} \geqslant e^{2}>k^{2}$ so that the convergence of $\sum_{r=0}^{\infty} k^{2 r} / p^{r}$ is assured. Further, since $g(n) \leqslant 1$ always,

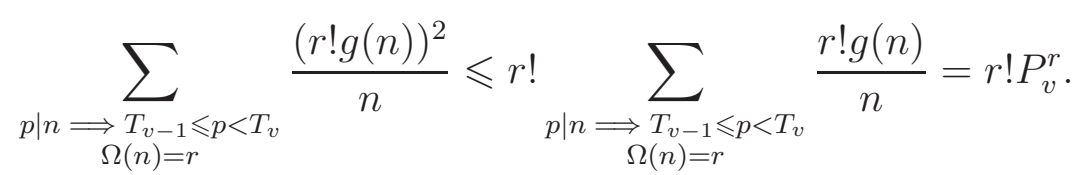

The second assertion of the proposition follows. 


\section{TWISTED FOURTH MOMENTS}

In order to establish Proposition 3 we shall require a formula for the twisted fourth moment,

$$
\int_{T}^{2 T}\left|\zeta\left(\frac{1}{2}+i t\right)\right|^{4} \cdot\left|\sum_{n \leqslant T^{\theta}} \frac{a(n)}{n^{1 / 2+i t}}\right|^{2} \Phi\left(\frac{t}{T}\right) d t
$$

where $\Phi$ is a smooth non-negative function such that $\Phi(x) \geqslant 1$ for $1 \leqslant x \leqslant 2$. Such mean values have been considered by many authors (for example see [7]), and we shall make use of the asymptotic established in [1].

To state the asymptotic formula, we introduce some notation. Put

$$
A_{z_{1}, z_{2}, z_{3}, z_{4}}=\frac{\zeta\left(1+z_{1}+z_{3}\right) \zeta\left(1+z_{1}+z_{4}\right) \zeta\left(1+z_{2}+z_{3}\right) \zeta\left(1+z_{2}+z_{4}\right)}{\zeta\left(2+z_{1}+z_{2}+z_{3}+z_{4}\right)},
$$

and

$$
B_{z_{1}, z_{2}, z_{3}, z_{4}}(n)=\prod_{p^{n_{p}} \| n}\left(\sum_{j \geqslant 0} \frac{\sigma_{z_{1}, z_{2}}\left(p^{n_{p}+j}\right) \sigma_{z_{3}, z_{4}}\left(p^{j}\right)}{p^{j}}\right)\left(\sum_{j \geqslant 0} \frac{\sigma_{z_{1}, z_{2}}\left(p^{j}\right) \sigma_{z_{3}, z_{4}}\left(p^{j}\right)}{p^{j}}\right)^{-1}
$$

where $\sigma_{z_{1}, z_{2}}(n)=\sum_{n_{1} n_{2}=n} n_{1}^{-z_{1}} n_{2}^{-z_{2}}$ and $n_{p}$ is the highest power of $p$ dividing $n$. Finally, define

$$
F\left(z_{1}, z_{2}, z_{3}, z_{4}\right)=A_{z_{1}, z_{2}, z_{3}, z_{4}} \sum_{m, n} \frac{a(n) \overline{a(m)}}{[m, n]} B_{z_{1}, z_{2}, z_{3}, z_{4}}\left(\frac{n}{(m, n)}\right) B_{z_{3}, z_{4}, z_{1}, z_{2}}\left(\frac{m}{(m, n)}\right) .
$$

Note that $F$ depends on the coefficients of the Dirichlet polynomial twisting the fourth moment.

Proposition 4. Let $T \geqslant 2$ and let $\Phi(x)$ be a smooth function supported on $[1 / 2,4]$ satisfying $\Phi^{(j)}(x) \ll_{\varepsilon} T^{\varepsilon}$ for any $j \geqslant 0$ and all $\varepsilon>0$. Let $a(n)$ be a sequence of complex numbers obeying the bound $|a(n)| \ll_{\varepsilon} n^{\varepsilon}$ for all $n \geqslant 1$ and all $\varepsilon>0$. Then, for $\theta<\frac{1}{4}$, we have

$$
\begin{aligned}
& \int_{\mathbb{R}}\left|\zeta\left(\frac{1}{2}+i t\right)\right|^{4} \cdot\left|\sum_{n \leqslant T^{\theta}} \frac{a(n)}{n^{1 / 2+i t}}\right|^{2} \Phi\left(\frac{t}{T}\right) d t=O\left(T^{1-\epsilon}\right)+ \\
& \quad \frac{1}{4(2 \pi i)^{4}} \int_{\substack{\left|z_{j}\right|=3^{j} / \log T \\
1 \leqslant j \leqslant 4}} F\left(z_{1}, z_{2}, z_{3}, z_{4}\right) \Delta\left(z_{1}, z_{2},-z_{3},-z_{4}\right)^{2}\left(\int_{\mathbb{R}} \Phi\left(\frac{t}{T}\right) \prod_{j=1}^{4}\left(\frac{t}{2 \pi}\right)^{z / 2} d t\right) \prod_{j=1}^{4} \frac{d z_{j}}{z_{j}^{4}}
\end{aligned}
$$

where

$$
\Delta\left(z_{1}, z_{2}, z_{3}, z_{4}\right)=\prod_{1 \leqslant i<j \leqslant 4}\left(z_{j}-z_{i}\right)
$$

denotes the Vandermonde determinant. 
Proof. Theorem 1 in [1] gives an asymptotic formula for

$$
\int_{\mathbb{R}} \zeta\left(\frac{1}{2}+\alpha_{1}+i t\right) \zeta\left(\frac{1}{2}+\alpha_{2}+i t\right) \zeta\left(\frac{1}{2}+\alpha_{3}-i t\right) \zeta\left(\frac{1}{2}+\alpha_{4}-i t\right)\left|\sum_{n \leqslant T^{\theta}} \frac{a(n)}{n^{1 / 2+i t}}\right|^{2} \Phi\left(\frac{t}{T}\right) d t
$$

with $\alpha_{1}, \alpha_{2}, \alpha_{3}, \alpha_{4}$ complex numbers of modulus $\ll(\log T)^{-1}$. We apply Lemma 2.5.1

of [3] to express that formula in terms of a multiple contour integral. Setting all the shifts $\alpha_{j}$ equal to zero then gives the claim.

\section{Proof of Proposition 3}

Again we confine ourselves to proving the second assertion of the proposition; the first statement follows similarly. We apply Proposition 4 with coefficients $a(n)$ given by

$$
\sum_{n} \frac{a(n)}{n^{s}}=\left(\prod_{2 \leqslant j<v} \mathcal{N}_{j}(s ; k-2)\right) \mathcal{P}_{v}(s)^{r},
$$

and taking $\Phi$ to be a non-negative smooth function supported on $[1 / 2,4]$ with $\Phi(x)=$ 1 on $[1,2]$. On the circles $\left|z_{j}\right|=3^{j} / \log T$ (for $1 \leqslant j \leqslant 4$ ) we note that

$$
\Delta\left(z_{1}, z_{2},-z_{3},-z_{4}\right)^{2} \ll(\log T)^{-12}, \quad A_{z_{1}, z_{2}, z_{3}, z_{4}} \ll(\log T)^{4},
$$

and that

$$
\int_{\mathbb{R}} \Phi\left(\frac{t}{T}\right) \prod_{j=1}^{4}\left(\frac{t}{2 \pi}\right)^{z_{j} / 2} d t \ll T
$$

Therefore by Proposition 4 we conclude that

$$
\int_{T}^{2 T}\left|\zeta\left(\frac{1}{2}+i t\right)\right|^{4}\left|\sum_{n} \frac{a(n)}{n^{1 / 2+i t}}\right|^{2} d t \ll T(\log T)^{4} \cdot \max _{\left|z_{j}\right|=3^{j} / \log T}\left|G\left(z_{1}, z_{2}, z_{3}, z_{4}\right)\right|
$$

where

$$
G\left(z_{1}, z_{2}, z_{3}, z_{4}\right)=\sum_{n, m} \frac{a(n) a(m)}{[n, m]} B_{z_{1}, z_{2}, z_{3}, z_{4}}\left(\frac{n}{(n, m)}\right) B_{z_{3}, z_{4}, z_{1}, z_{2}}\left(\frac{m}{(n, m)}\right) .
$$

The estimate in Proposition 3 will now follow once we establish the bound

$$
G\left(z_{1}, z_{2}, z_{3}, z_{4}\right) \ll\left(\log T_{v-1}\right)^{k^{2}-4}\left(18^{r} r ! P_{v}^{r} \exp \left(P_{v}\right)\right),
$$

when $\left|z_{j}\right|=3^{j} / \log T$ for $1 \leqslant j \leqslant 4$. 
From the multiplicative nature of the coefficients $a$, and $B_{z_{1}, z_{2}, z_{3}, z_{4}}$, we may express $G\left(z_{1}, z_{2}, z_{3}, z_{4}\right)$ as the product of

$$
\prod_{2 \leqslant j<v}\left(\sum_{\substack{p \mid n, m) \\ \Omega(n), \Omega(m) \leqslant 500 P_{j}}} \frac{(k-2)^{\Omega(n)+\Omega(m)} g(n) g(m)}{[n, m]} B_{z_{1}, z_{2}, z_{3}, z_{4}}\left(\frac{n}{(m, n)}\right) B_{z_{3}, z_{4}, z_{1}, z_{2}}\left(\frac{m}{(m, n)}\right)\right),
$$

and

$$
\sum_{\substack{p \mid m n \\ \Omega(m)=\Omega(n)=r}} \frac{r !^{2} g(m) g(n)}{[m, n]} B_{z_{1}, z_{2}, z_{3}, z_{4}}\left(\frac{n}{(n, m)}\right) B_{z_{3}, z_{4}, z_{1}, z_{2}}\left(\frac{m}{(m, n)}\right) .
$$

We now estimate the quantities in (5) and (6). To do this, it is helpful to note that from the definition (2) one has for $p \leqslant T^{10^{-8}}$ and $\left|z_{j}\right|=3^{j} / \log T$

$$
B_{z_{1}, z_{2}, z_{3}, z_{4}}\left(p^{u}\right)=\sigma_{z_{1}, z_{2}}\left(p^{u}\right)\left(1+O\left(\frac{1}{p}\right)\right),
$$

from which we may deduce that

$$
\left|B_{z_{1}, z_{2}, z_{3}, z_{4}}(n)\right| \ll d_{3}(n) \leqslant 3^{\Omega(n)},
$$

for integers $n$ composed only of primes below $T^{10^{-8}}$, and where $d_{3}$ denotes the 3 divisor function.

Consider first the expression in (6). Using (8) we have $\left|B_{z_{1}, z_{2}, z_{3}, z_{4}}(n /(n, m))\right| \ll 3^{r}$ and $\left|B_{z_{3}, z_{4}, z_{1}, z_{2}}(m /(n, m))\right| \ll 3^{r}$, and so the quantity in (6) is

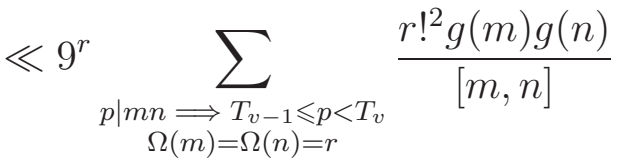

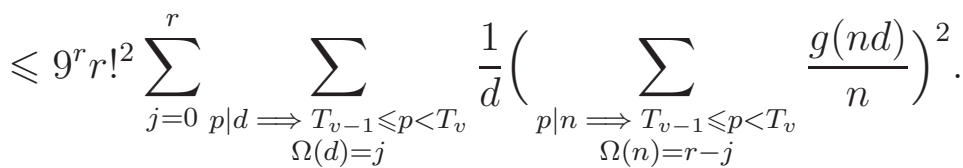

Since $g(n d) \leqslant g(n)$, the above may be bounded by

$$
\leqslant 9^{r} r !^{2} \sum_{j=0}^{r}\left(\frac{1}{j !} P_{v}^{j}\right)\left(\frac{1}{(r-j) !} P_{v}^{r-j}\right)^{2}=9^{r} r ! P_{v}^{r} \sum_{j=0}^{r}\left(\begin{array}{l}
r \\
j
\end{array}\right) \frac{P_{v}^{r-j}}{(r-j) !} \leqslant 18^{r} r ! P_{v}^{r} \exp \left(P_{v}\right),
$$

upon noting that $\left(\begin{array}{l}r \\ j\end{array}\right) \leqslant 2^{r}$ and $\sum_{j=0}^{r} P_{v}^{r-j} /(r-j) ! \leqslant \exp \left(P_{v}\right)$.

Now we turn to the expression in (5), treating the contribution for a given $j$ in the range $2 \leqslant j<v$. First we show that the constraints $\Omega(n)$ and $\Omega(m) \leqslant 500 P_{j}$ may be dropped from the expression there with negligible error. We bound these terms 
using Rankin's trick, in the form $\exp \left(\Omega(m)+\Omega(n)-500 P_{j}\right) \geqslant 1$ if either $\Omega(m)$ or $\Omega(n)$ exceeds $500 P_{j}$. By $(8)$ and since $|k-2| \leqslant 2$, the error induced in dropping the constraint on $\Omega(m)$ and $\Omega(n)$ is

$$
\begin{aligned}
& \leqslant e^{-500 P_{j}} \sum_{p \mid m, n} \sum_{T_{j-1} \leqslant p<T_{j}} \frac{(2 e)^{\Omega(m)+\Omega(n)}}{[m, n]} d_{3}(m) d_{3}(n) \\
& \ll e^{-500 P_{j}} \prod_{T_{j-1} \leqslant p<T_{j}}\left(1+\frac{6 e+6 e+(6 e)^{2}}{p}+O\left(\frac{1}{p^{2}}\right)\right) \ll e^{-100 P_{j}} .
\end{aligned}
$$

After discarding the constraint on $\Omega(m)$ and $\Omega(n)$, the contribution of the term in (5) is

$$
\prod_{T_{j}-1 \leqslant p<T_{j}}\left(\sum_{a, b=0}^{\infty} \frac{(k-2)^{a+b} g\left(p^{a}\right) g\left(p^{b}\right)}{p^{\max (a, b)}} B_{z_{1}, z_{2}, z_{3}, z_{4}}\left(p^{a-\min (a, b)}\right) B_{z_{3}, z_{4}, z_{1}, z_{2}}\left(p^{b-\min (a, b)}\right)\right) .
$$

Upon using (7), we see that only the terms $a, b=0$, or 1 are relevant and the total contribution is

$$
\begin{aligned}
& \prod_{T_{j-1} \leqslant p<T_{j}}\left(1+\frac{(k-2)\left(\sigma_{z_{1}, z_{2}}(p)+\sigma_{z_{3}, z_{4}}(p)\right)+(k-2)^{2}}{p}+O\left(\frac{1}{p^{2}}\right)\right) \\
= & \prod_{T_{j-1} \leqslant p<T_{j}}\left(1+\frac{k^{2}-4}{p}+O\left(\frac{1}{p^{2}}+\frac{\log p}{p \log T}\right)\right),
\end{aligned}
$$

since $\sigma_{z_{1}, z_{2}}(p)=p^{-z_{1}}+p^{-z_{2}}=2+O(\log p / \log T)$, and similarly for $\sigma_{z_{3}, z_{4}}(p)$. We conclude that the expression in (5) equals

$$
\prod_{2 \leqslant j<v}\left(\prod_{T_{j-1} \leqslant p<T_{j}}\left(1+\frac{k^{2}-4}{p}+O\left(\frac{1}{p^{2}}+\frac{\log p}{p \log T}\right)\right)+O\left(e^{-100 P_{j}}\right)\right) \ll\left(\log T_{v-1}\right)^{k^{2}-4} .
$$

Combining this estimate with (9), the bound (4) follows, and with it the proof of Proposition 3 is complete.

\section{REFERENCES}

[1] S. Bettin, H. M. Bui, X. Li, M. Radziwitł, A quadratic divisor problem and moments of the Riemann zeta-function, preprint, available at arXiv.1609.02539.

[2] S. Bettin, V. Chandee, M. Radziwiłl, The mean square of the product of $\zeta(s)$ with Dirichlet polynomials, J. Reine Angew. Math, 729 (2017), 51-79.

[3] J. B. Conrey, D. W. Farmer, J. P. Keating, M. O. Rubinstein, N. C. Snaith, Integral moments of $L$-functions, Proc. London Math. Soc. 91 no. 3 (2005), 33-104

[4] A. Harper, Sharp conditional bounds for moments of the Riemann zeta function. Preprint available at arXiv.1305.4618. 
[5] D. R. Heath-Brown, Fractional moments of the Riemann zeta function, J. London Math. Soc., 24, no. 1 (1981), 65-78.

[6] G.H. Hardy, J.E. Littlewood, Contributions to the theory of the Riemann zeta-function and the theory of the distribution of primes, Acta Arith. 41 (1918), 119-196.

[7] C. P. Hughes, M. P. Young, The twisted fourth moment of the Riemann zeta function, J. Reine Angew. Math. 641 (2010), 203-236.

[8] A. E. Ingham, Mean-value theorems in the theory of the Riemann zeta function, Proc. London Math. Soc. 27 (1926), 273-300.

[9] M. Radziwiłł, K. Soundararajan, Continuous lower bounds for moments of zeta and L-functions, Mathematika, 59 no. 1 (2013), 119-128.

[10] M. Radziwiłł, K. Soundararajan, Moments and distribution of central L-values of quadratic twists of elliptic curves, Invent. Math. 202 no. 3 (2015) 1029-1068

[11] K. Ramachandra, Some remarks on the mean value of the Riemann zeta function and other Dirichlet series, Ann. Acad. Sci. Fennicae, 5 (1980), 145-158

[12] K. Ramachandra. Some remarks on the mean value of the Riemann zeta function and other Dirichlet series. II Hardy-Ramanujan J., 3 (1980), 1-24.

[13] K. Soundararajan, Moments of the Riemann zeta function, Annals of Math. 170 no. 2 (2009), 981-993.

Department of Mathematics, University College London, 25 Gordon Street, LonDON WC1H.

E-mail address: winstonheap@gmail.com

Department of Mathematics Caltech, 1200 E California Blvd Pasadena, CA, 91125

E-mail address: maksym.radziwill@gmail.com

Department of Mathematics, Stanford University, Stanford, CA 94305, USA.

E-mail address: ksound@stanford.edu 